\title{
Hashimoto's thyroiditis-induced cryptogenic organizing pneumonia: A case report
}

\author{
LITAO GUO $^{1^{*}}, \mathrm{BO} \mathrm{CHEN}^{2 *}, \mathrm{LEI} \mathrm{ZHANG}^{1}, \mathrm{YUAN} \mathrm{DENG}^{3}, \mathrm{HAO} \mathrm{LI}^{1}$ and QIN DONG SHI ${ }^{1}$ \\ ${ }^{1}$ Department of Critical Care Medicine, The First Affiliated Hospital of Xi'an Jiaotong University, Xi'an, Shaanxi 710061; \\ ${ }^{2}$ Department of Critical Care Medicine, Shangluo Central Hospital, Shangluo, Shaanxi 726000; ${ }^{3}$ Department of Pathology, \\ The First Affiliated Hospital of Xi'an Jiaotong University, Xi'an, Shaanxi 710061, P.R. China
}

Received February 27, 2019; Accepted September 19, 2019

DOI: $10.3892 /$ etm.2019.8143

\begin{abstract}
A 49-year-old female patient developed chest tightness and shortness of breath without apparent cause and presented to a local hospital. Chest radiography indicated increased thickening of the lung texture, increased multiple patchy densities in the lower lobes of the bilateral lungs and a slightly enlarged thyroid. The patient was treated for pulmonary infection with antibiotics but the symptoms persisted. A repeated CT scan revealed ground-glass attenuation of the bilateral lungs with multiple flaky exudates and visible bronchogenic signs. The symptoms did not improve after broadening anti-microbial coverage. Bronchoscopy and biopsy confirmed cryptogenic organizing pneumonia (COP). Thyroid ultrasound and thyroid function tests jointly confirmed the diagnosis of Hashimoto's thyroiditis (HT). After receiving corticosteroid treatment, the patient's condition improved and she was discharged. This case demonstrates that COP may present in combination with Hashimoto's thyroiditis (HT) and may possibly even be caused by HT. Early confirmation of diagnosis and treatment will help to improve the prognosis of such patients.
\end{abstract}

\section{Introduction}

Cryptogenic organizing pneumonia (COP), also known as bronchiolitis obliterans with organizing pneumonia (BOOP), occurs without obvious pathogens or other concomitant diseases. It presents as lesions involving mainly the alveolar spaces, alveolar ducts, small airways and interstitial lung.

Correspondence to: Professor Qin Dong Shi, Department of Critical Care Medicine, The First Affiliated Hospital of Xi'an Jiaotong University, 277 West Yanta Road, Xi'an, Shaanxi 710061, P.R. China

E-mail: shiqindong@163.com

${ }^{*}$ Contributed equally

Key words: cryptogenic organizing pneumonia, Hashimoto's thyroiditis, bronchiolitis obliterans with organizing pneumonia
Histopathological findings demonstrate the proliferation of granulation tissue in the alveolar spaces and bronchioles. Clinical manifestations include interstitial lung disease with restrictive ventilatory dysfunction $(1,2)$. In 1983, Davision et al (3) referred to as-yet undescribed BOOP as COP. In 2013, the American Thoracic Society and the European Respiratory Society reclassified idiopathic interstitial pneumonia (IIP) into three categories, i.e., main, rare and unclassifiable; COP is categorised as main IIP (4). The incidence of COP is low. It is sensitive to glucocorticoids and has a good prognosis. However, COP presents with no specific clinical manifestations and imaging features, lacks specific biomarkers and is particularly difficult to distinguish from community-acquired pneumonia.

Genetic, environmental, immune and other pathogenic factors, including viral infections, interact to maintain a high incidence of thyroid disease (5). Among these, autoimmune thyroid diseases account for $\sim 90 \%$ of thyroid diseases (6). Hashimoto's thyroiditis (HT), also known as chronic lymphocytic thyroiditis, is a classic type of autoimmune thyroiditis. It usually manifests as a local inflammatory response and is closely linked to autoimmunity. The disease was first reported by the Japanese scholar Hakaru Hashimoto in 1912 (7), and is thus also known as Hashimoto's disease. Its pathogenesis is mediated by a large number of lymphocytes that infiltrate the thyroid parenchyma, repeatedly destroying the follicular structure of the gland and causing hyperplasia of the interstitial fibres. This results in diffuse or uneven nodularity. HT is accompanied by thyroid tumours (8-11) and hypothyroidism but rarely causes COP. It has been reported that the prevalence of HT with papillary thyroid carcinoma is $0.5-38 \%$ (10). Studies have also indicated that HT is an important risk factor for primary non-Hodgkin's lymphoma (12), and it has even been suggested that HT should be considered a pre-cancerous precursor of thyroid cancer.

In the available literature, HT combined with COP has been reported infrequently (13). One case series reporting on 18 cases of organizing pneumonia indicated that it frequently occurred concomitantly with infections and autoimmune disease. One of the 18 patients had organizing pneumonia that was considered to have been caused by Hashimoto disease (13). HT as well as COP are associated with viral infection and autoimmunity, and there are certain similar risk factors. Therefore, 
patients with Hashimoto's thyroiditis complicated by concurrent COP may be more seriously ill and require longer hospital stays. Whether long-term follow-up is able to detect a higher incidence of thyroid tumours is currently elusive and warrants further investigation.

\section{Case report}

Case presentation. A 49-year-old female developed chest tightness and shortness of breath without apparent cause and presented at a local hospital. Chest radiographs indicated increased thickening of the lung texture and increased multiple patchy densities in the lower lobes of the bilateral lungs, and a slightly increased thyroid was seen on examination. The patient underwent electrocardiogram (ECG) and echocardiography to exclude cardiogenic factors, was diagnosed with "pulmonary infection, goitre' and given antibiotic treatment with levofloxacin and ribavirin for 9 days. The patient's symptoms were not significantly relieved and the shortness of breath gradually worsened, with associated fever, cough and a small amount of white sticky sputum. Chest CT imaging indicated 'pulmonary infection' and the antibiotics were broadened to meropenem, vancomycin and fluconazole. After this treatment, the patient's shortness of breath worsened progressively and she remained febrile with a maximum temperature of $38.7^{\circ} \mathrm{C}$. After 5 days of treatment, chest CT indicated that the lung exudates were significantly increased. The patient was transferred from the local hospital to the critical care unit at The First Affiliated Hospital of Xi'an Jiaotong University. The patient was conscious on admission, with low body weight $(49 \mathrm{~kg})$. The patient's vital signs included a body temperature of $37.2^{\circ} \mathrm{C}$, heart rate of 97 beats $/ \mathrm{min}$, respiratory rate of 37 breaths/min and blood pressure of 112/69 $\mathrm{mmHg}$. On examination, thyroid enlargement (grade 2) was noted. The thyroid was firm and vascular murmur was absent. The lungs exhibited dullness with percussion, and moist rales were heard at the bilateral lung bases.

The patient was admitted to the hospital and subsequently, her respiratory status further deteriorated. Arterial blood gas analysis demonstrated the following: $\mathrm{pH} 7.42$; oxygen partial pressure, $54.0 \mathrm{mmHg}$; carbon dioxide partial pressure, $35.7 \mathrm{mmHg}$. These findings were consistent with Type 1 respiratory failure and the patient was put on non-invasive mechanical ventilation. Chest CT indicated ground-glass changes in the bilateral lungs and multiple large high-density lesions. The C-reactive protein level was $65.6 \mathrm{mg} / \mathrm{l}$ and a diagnosis of severe pneumonia was considered. The patient received a combination broad-spectrum anti-microbial treatment with imipenem, moxifloxacin, ganciclovir and voriconazole. After one week of treatment, the patient still had difficulty breathing and was not able to be weaned from the non-invasive ventilator; the patient was still experiencing intermittent fever with a body temperature of up to $38.6^{\circ} \mathrm{C}$, without any significant decrease in C-reactive protein levels. Bronchoscopy with alveolar lavage and lung biopsy was performed in order to exclude any infectious or malignant aetiologies for the patient's respiratory symptoms.

Assessments. The initial and subsequent complete blood cell counts revealed low and fluctuating haemoglobin, a normal to low white blood cell count, elevated neutrophil percentage, as well as normal platelets and eosinophils. Liver function indicators were within normal ranges except for serum sodium, which was low. Serum inflammatory markers (C-reactive protein and erythrocyte sedimentation rate) were elevated. The patient's coagulation profile indicated elevated activated partial thromboplastin time, fibrinogen content and D-dimer. All values are provided in Table I.

Initial chest radiographs indicated increased thickening of the lung texture, increased multiple patchy densities in the lower lobes of the bilateral lungs and a small bilateral pleural effusion (Fig. 1). After the patient was admitted to the intensive care unit of our hospital, non-invasive mechanical ventilation was provided. Chest CT revealed decreased translucency and increased ground-glass changes in the bilateral lung fields, with multiple large high-density lesions obscuring the borders of the lobar fissures. Air bronchograms were observed within a large high-density lesion (Fig. 2A-C).

Bronchoscopy revealed mild bilateral bronchial oedema but no masses, haemorrhage or stenosis that may have suggested inflammation of the bronchial mucosa. Examination of the bronchoalveolar lavage fluid indicated negativity on tuberculosis smears, tuberculosis DNA test, bacterial and fungal smears, and bacterial and fungal cultures. Blood and lavage fluids were negative for tumour-associated markers. Cytological examination of lavage fluid revealed no malignant cells, but columnar cells, squamous cells, alveolar macrophages, lymphocytes and neutrophils were observed (Fig. 3). The diagnoses of systemic lupus erythematosus and sarcoidosis were not supported, and all of the above results did not support a diagnosis of bacterial, fungal, viral or atypical pathogenic infections, including pneumocystis.

The lung biopsy revealed characteristic changes of COP, with loose fibroblast plugs in the alveolar space and extensive proliferation of fibroblasts (Fig. 4). Eosinophils were not observed in the lung biopsy and Churg-Strauss syndrome was ruled out.

Blood and urine cultures and urine smears were negative. Procalcitonin and 1,3- $\beta$-d-glucan were normal and galactomannan was elevated (Table I). Tests for Epstein-Barr virus, cytomegalovirus, human immunodeficiency virus, hepatitis B virus DNA, hepatitis and respiratory syncytial virus antibodies were all negative, as were the antigen tests for Streptococcus pneumoniae, Mycoplasma pneumoniae, Toxoplasma gondii, Legionella pneumophila and Treponema pallidum.

The rheumatology work-up was negative for perinuclear anti-neutrophil cytoplasmic antibodies, cytoplasmic anti-neutrophil antibodies and double-stranded DNA antibodies. Anti-nuclear antibodies (1:80) were weakly positive, a non-specific finding. The percentage of killer $\mathrm{T}$ cells $\left(\mathrm{CD}^{+} \mathrm{CD}^{+}\right)$was slightly elevated and the $\mathrm{CD} 4 / \mathrm{CD} 8$ ratio was low (Table I).

Thyroid ultrasound revealed a diffusely enlarged symmetric thyroid gland with enlargement of the isthmus, a smooth and well-demarcated capsule, hypoechoic nodules visible in the bilateral lobes and regular morphology. Colour Doppler imaging indicated slightly increased vascularity of the gland. Thyroid function tests revealed markedly increased thyroid peroxidase auto-antibody (TPOAb), low triiodothyronine, normal free thyroxine, elevated thyroid stimulatory hormone and normal thyroglobulin antibody (TgAb) (Table I). 
Table I. Summary of diagnostic indicators for the patient.

A, Blood cell count

\begin{tabular}{lccc}
\hline Variable & Patient value & Normal range $^{\mathrm{a}}$ & $\begin{array}{c}\text { Characterization of patient } \\
\text { level compared with normal level }\end{array}$ \\
\hline Haemoglobin $(\mathrm{g} / \mathrm{l})$ & 85 & $110-150$ & Low \\
White cell count $\left(\mathrm{x} 10^{9} / \mathrm{l}\right)$ & 5.93 & $3.5-9.5$ & Normal to low \\
Neutrophils $(\%)$ & 79.8 & $40-75$ & Elevated \\
Platelets $\left(\mathrm{x} 10^{9} / \mathrm{l}\right)$ & 280 & $125-350$ & Normal \\
Eosinophils $\left(\mathrm{x} 10^{9} / \mathrm{l}\right)$ & 0.11 & $0.02-0.52$ & Normal \\
\hline
\end{tabular}

B, Liver function

\begin{tabular}{lccc} 
Variable & Patient value & Normal range & $\begin{array}{c}\text { Characterization of patient } \\
\text { level compared with normal level }\end{array}$ \\
\hline $\mathrm{Ca}(\mathrm{mmol} / \mathrm{l})$ & 2.06 & $2.11-2.52$ & Low \\
$\mathrm{Mg}(\mathrm{mmol} / \mathrm{l})$ & 0.94 & $0.75-1.02$ & Normal \\
$\mathrm{Urea}(\mathrm{mmol} / \mathrm{l})$ & 2.46 & $2.6-7.5$ & Low \\
Creatinine $(\mu \mathrm{mol} / \mathrm{l})$ & 46 & $41-73$ & Normal \\
Serum Na $(\mathrm{mmol} / \mathrm{l})$ & 131 & $137-147$ & Low \\
\hline
\end{tabular}

C, Inflammatory markers

Characterization of patient

Variable

Patient value

Normal range

65.6

0.0-10.0

0.0-20

level compared with normal level

C-reactive protein $(\mathrm{mg} / \mathrm{l})$

Erythrocyte sedimentation rate $(\mathrm{mm} / \mathrm{h})$

91

Elevated

Elevated

D, Coagulation profile

Characterization of patient

Variable

Patient value

Normal range

level compared with normal level

Activated partial thromboplastin time (sec)

Fibrinogen content (g/l)

45

5.69

1.14

$28-43.5$
$2.0-4.0$
$0-1$

$0-1$
Elevated

Elevated

Elevated

E, Rheumatology

Variable

Anti-nuclear antibodies (ratio)

$\mathrm{CD}^{+}{ }^{+} \mathrm{CD}^{+}{ }^{+}$Killer T-cells $(\%)$

CD4/CD8

Procalcitonin $(\mathrm{ng} / \mathrm{ml})$

1-3-b-D glucans (pg/ml)

Galactomannan $(\mu \mathrm{g} / \mathrm{l})$
Patient value

$1: 80$
31.93
0.58
0.043
$<10$
0.96

Normal range

$\leq 1: 80$
$18.1-29.6$
$0.8-2.1$
$<0.5$
$<60$
$<0.5$

Characterization of patient level compared with normal level
Normal
Elevated
Low
Normal
Normal
Elevated

\section{F, Thyroid function}

Characterization of patient

Variable

Patient value

Normal range

level compared with normal level

$\begin{array}{lccc}\mathrm{TPOAb}(\mathrm{U} / \mathrm{ml}) & >3,000 & <15 & \text { Greatly elevated } \\ \mathrm{T}_{3}(\mathrm{ng} / \mathrm{ml}) & 0.660 & 0.78-2.20 & \text { Low }\end{array}$


Table I. Continued.

\begin{tabular}{lccc} 
Variable & Patient value & Normal range & $\begin{array}{c}\text { Characterization of patient } \\
\text { level compared with normal level }\end{array}$ \\
\hline $\mathrm{FT}_{4}(\mathrm{pmol} / \mathrm{l})$ & 7.96 & $9.05-25.5$ & Low \\
$\mathrm{TSH}(\mathrm{uIU} / \mathrm{ml})$ & 9.61 & $0.25-5$ & Elevated \\
$\mathrm{TgAb}(\%)$ & 22.1 & $<30$ & Normal \\
\hline
\end{tabular}

TPOAb, thyroid peroxidase autoantibody; $\mathrm{Tg}$, thyroglobulin; $\mathrm{TSH}$, thyroid-stimulating hormone; $\mathrm{FT}_{4}$, free thyroxine; $\mathrm{T}_{3}$, tri-iodothyronine ${ }^{a}$ The normal range value data was supplied by the Analytical Laboratories of The First Affiliated Hospital of Xi'an Jiaotong University, China.

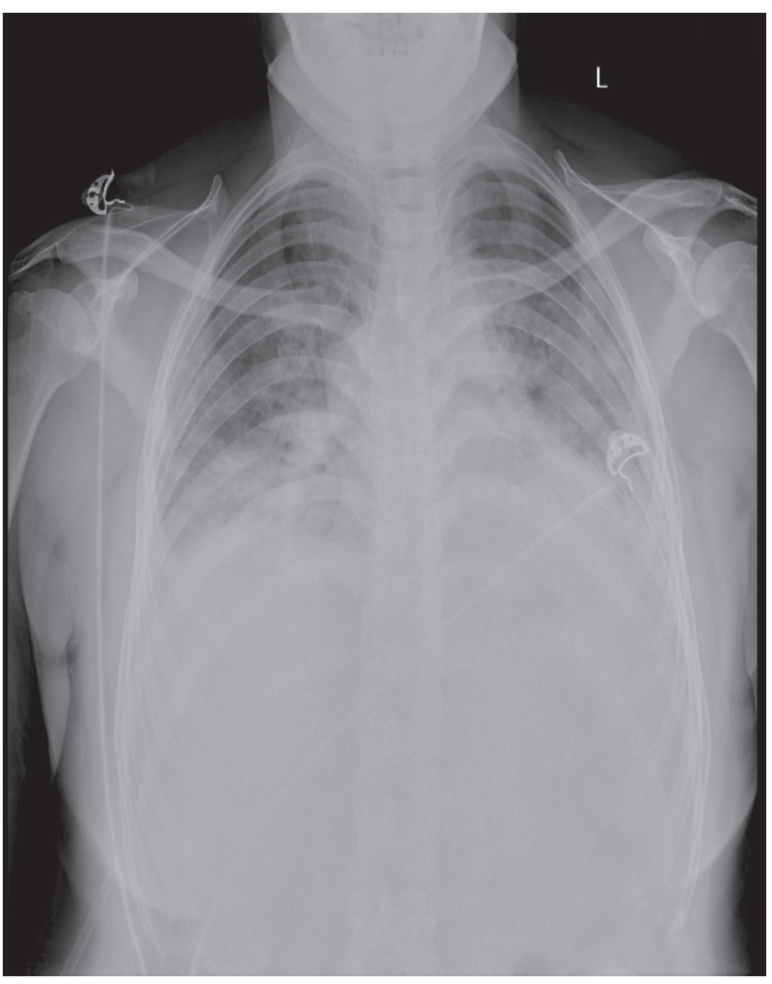

Figure 1. Chest radiography indicated increased thickening of the lung texture, increased multiple patchy densities in the lower lobes of the bilateral lungs and a small bilateral pleural effusion.

Differential diagnosis. The early onset of the patient's respiratory symptoms and early tests led us to consider bacterial pneumonia, fungal pneumonia or rare pathogens. However, subsequent testing for these infections and extensive tests mentioned above were all negative. The patient received antibiotic treatment directly after admission to the critical care unit, mainly due to her clinical symptoms and associated imaging.

It is noteworthy that anti-microbial therapy in patients with COP has not led to any improvement in pulmonary status. The cause of COP itself remains elusive. Conditions including aspneumonia, drug toxicity, lung cancer and non-specific interstitial pneumonia may cause COP-like pathological pulmonary symptoms.

It has been reported that the presence of multiple migratory patchy opacities seen on CT in the bilateral lungs is a characteristic radiographic manifestation of COP, and the area of lung bearing tho lesions may reflect disease severity to a certain extent (14). However, recent studies have indicated that conditions including hypersensitivity pneumonitis, eosinophilic pneumonia and fungal infections may present with similar radiographic patterns. It is difficult to diagnose COP from the clinical manifestations and imaging alone, particularly in the early stages of COP, given the wide variety of differential diagnoses encompassing numerous mimicking conditions (15).

Rheumatological causes, including systemic lupus erythematosus, sarcoidosis, polymyositis and Churg-Strauss syndrome, were also considered, but the blood work and lung biopsy were not suggestive of these aetiologies. Malignancy, including lymphoma or leukaemia, was also considered, and a bone marrow biopsy was performed to exclude these diagnoses.

HT may be distinguished from subacute thyroiditis due to its association with autoimmunity. HT frequently presents with clinical hypothyroidism and elevated TPOAb and TgAb. Subacute thyroiditis is associated with viral infections and is self-limited, usually without any long-term sequelae. HT is easy to confuse with certain types of thyroid tumour (8-11), requiring careful diagnostic attention.

Diagnostic focus was initially centred on the imaging features in the lungs and various pulmonary infections were carefully considered as the causative agent. The final diagnosis of COP was achieved with lung biopsies and histopathologic review. The patient's condition improved with corticosteroid treatment. Subacute thyroiditis requires regular review and follow-up for recurrence, rather than a particular treatment.

Treatment. Blood gas analysis of the patient indicated type I respiratory failure, which was treated with non-invasive mechanical ventilation. According to the chest CT findings and the patient's clinical presentation, severe pneumonia was considered to be the most likely diagnosis, even though the specific causative pathogen was not clear. The patient received broad-spectrum anti-microbial treatment with imipenem, moxifloxacin, ganciclovir and voriconazole for the treatment of the suspected pneumonia. The patient also received human intravenous immunoglobulin $0.4 \mathrm{~g} / \mathrm{kg} /$ day for 5 days.

During the course of treatment, there was no significant improvement seen on chest CT examinations. Testing for all associated pathogens was negative, and infection-associated indices, including white blood cell count and plateletcrit, were normal, yet the symptoms of the patient's clinical condition, including chest tightness and shortness of breath, did not 
A
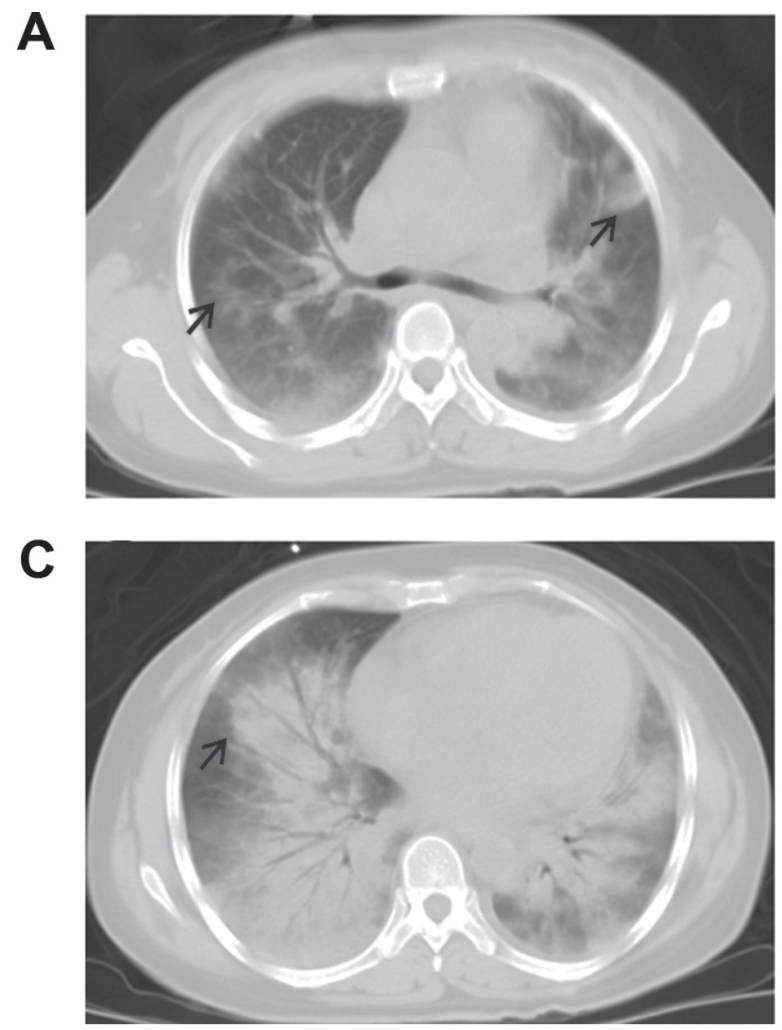

B

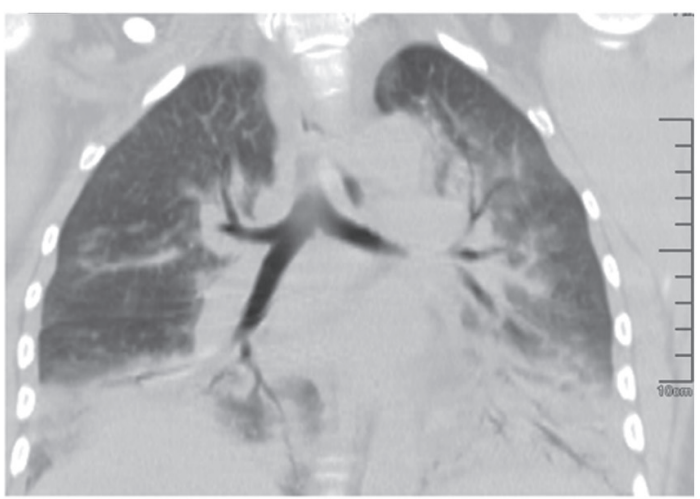

Figure 2. Chest CT: (A) Bilateral lung fields with reduced transmittance, ground glass changes and multiple large, high-density lesions in the bilateral lungs. (B) The middle and lower lobes of the lungs are indistinct with blurred boundaries. (C) Air bronchograms visible within a large high-density lesion.

improve. At one week after the start of anti-microbial therapy, fibrobronchoscopy with lung biopsy indicated characteristic changes of COP on histopathological analysis, thus establishing a diagnosis of COP.

After the definitive diagnosis, antibiotics were discontinued, and intravenous methylprednisolone ( $80 \mathrm{mg}$ daily) was initiated. After 5 days of treatment with methylprednisolone, the dose was reduced to $40 \mathrm{mg}$ daily. At this point, the patient's body temperature returned to normal, her shortness of breath gradually improved and the non-invasive ventilator was intermittently disengaged. After a week of treatment, chest $\mathrm{CT}$ indicated that the lung consolidations were decreased (Fig. 5), the shortness of breath and other symptoms resolved and the non-invasive ventilator was completely disengaged. The patient was discharged and continued treatment with oral prednisone $(0.75 \mathrm{mg} / \mathrm{kg} /$ day $)$, with a gradual reduction in symptoms noted on regular follow-up that was completed at 6 months. As HT presents with no specific symptoms, after excluding the possibility of thyroid tumours, it was decided not to provide any treatment, and to perform regular follow-up with thyroid ultrasound and thyroid function tests.

Outcome and follow-up. After 1 week of methylprednisolone treatment, the chest CT and radiograph indicated the gradual absorption of lung exudates and the symptoms of the patient were markedly improved, with no shortness of breath or fever. The serum white blood cell count and C-reactive protein were normal. The patient's lung function was normal at the 6- and 12-month follow-up visits and the chest CT indicated complete resolution of the previously seen opacities (Fig. 6). The patient demonstrated no further symptoms, including

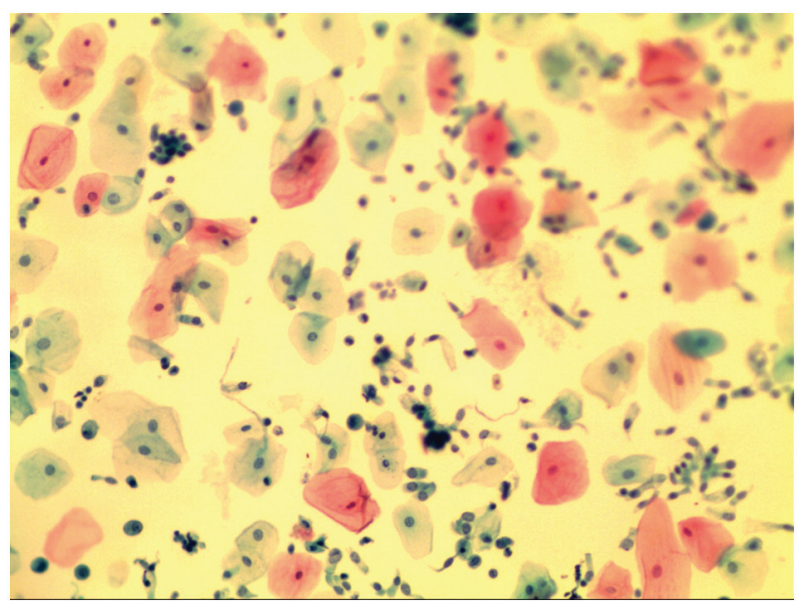

Figure 3. Lavage fluid cytological examination revealed no malignant cells, columnar cells, squamous cells, alveolar macrophages, lymphocytes or neutrophils (Pasteur; original magnification, x10).

shortness of breath, and her activities of daily life were not affected. The size of the thyroid gland was essentially normal and no obvious abnormalities were observed on ultrasound or laboratory assessment of thyroid function.

\section{Discussion}

COP occurs most frequently in 40-60-year-old individuals and occasionally in patients aged $<40$ years. Its onset is usually subacute or acute. Symptoms typically comprise respiratory complaints, including cough and dyspnoea (particularly with 

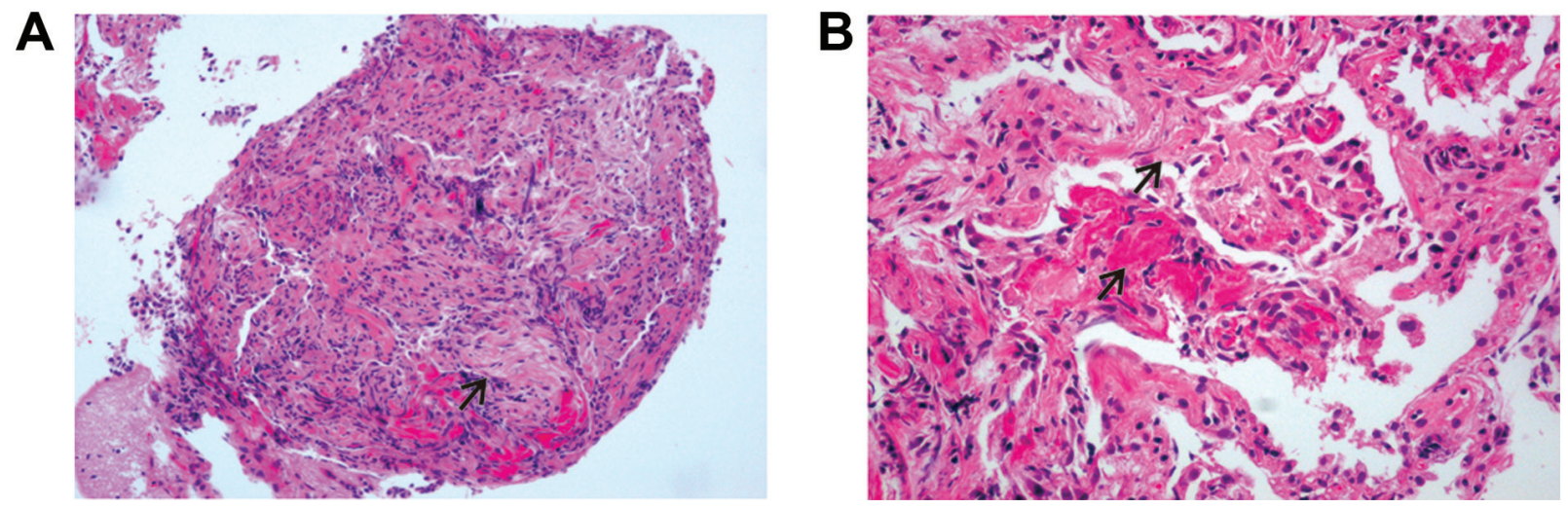

Figure 4. (A) Biopsy of bronchial tissue in the posterior segment of the lower lobe of the left lung revealed inflammation of the lung tissue and the presence of inflammatory cells in the alveolar lumina and septa. Fibrosis was detected in the alveolar septa and the small airways and is indicated with arrows (H\&E, original magnification, x10). (B) A higher magnification view of the biopsy image. Obliterative bronchiolitis and intraluminal fibroplasia are indicated with arrows (H\&E, original magnification, $\mathrm{x} 20$ ).
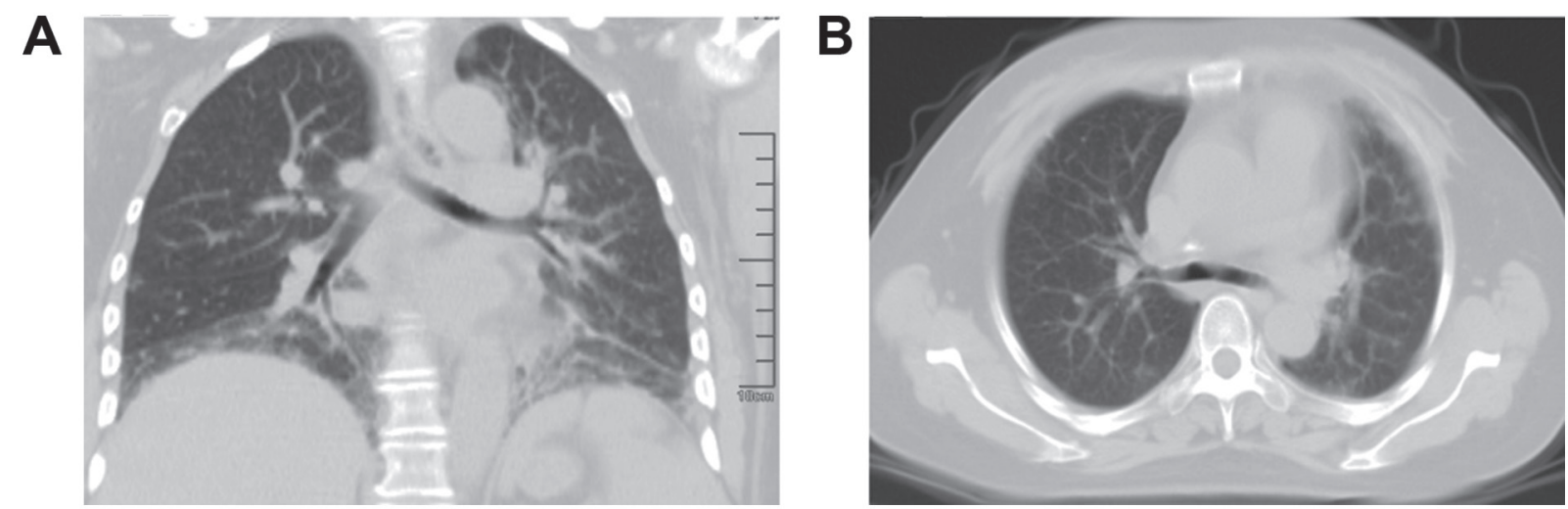

Figure 5. Chest CT scan after 1 month. (A) Longitudinal section, tracheal bifurcation plane: the exudation of both lungs gradually decreased and the transmittance was almost normal. (B) Cross section, tracheal bifurcation plane: Gradual resolution of lung consolidations.
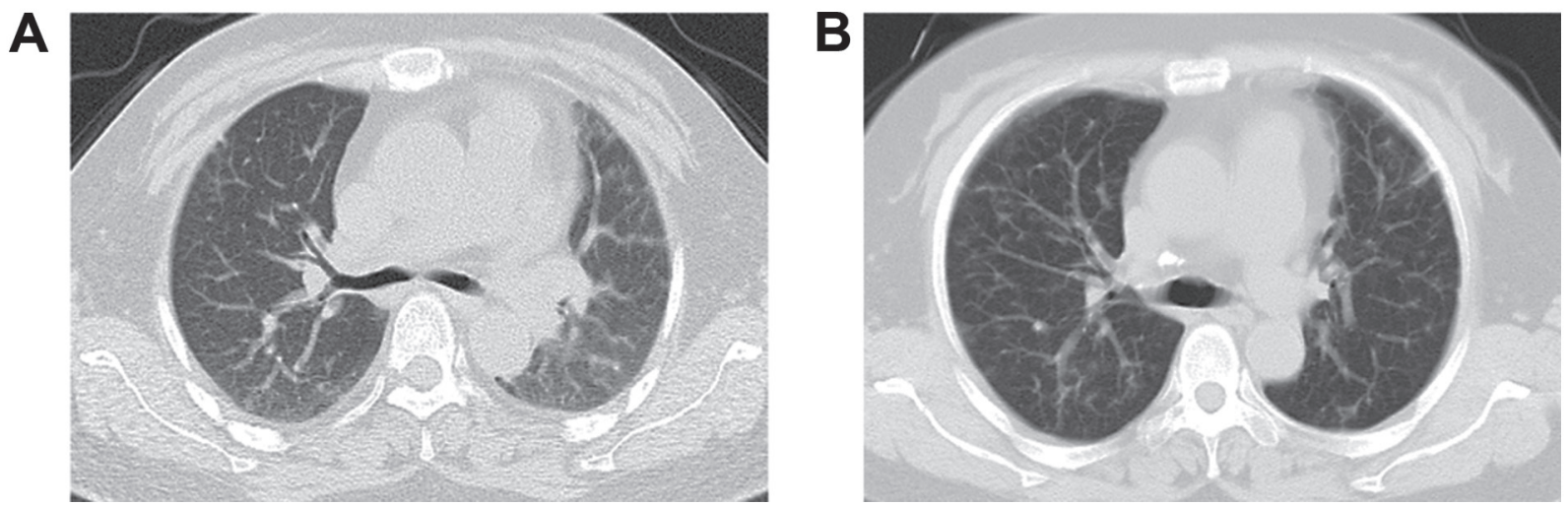

Figure 6. (A) CT scan after 6 months and (B) CT scan after 12 months, demonstrating no recurrence of cryptogenic organizing pneumonia.

exertion), and are frequently accompanied by fever, fatigue, weight loss and other systemic symptoms (16). Physical examination may at times reveal wet rales on auscultation of the lungs. Common laboratory abnormalities include elevated white blood cell count, erythrocyte sedimentation rate and C-reactive protein levels $(17,18)$. Pulmonary function testing may indicate mild to moderate restrictive ventilatory dysfunction, with or without reduced diffusion capacity, and blood gas analysis may suggest mild hypoxaemia. Chest radiographs demonstrate a similar appearance to pneumonia, with no clear characteristic changes of COP. CT may indicate unilateral or bilateral opacities, mostly in the subpleural and peribronchial regions, and may also demonstrate air bronchograms, ground-glass attenuation or pulmonary nodules with rare isolated nodules displaying as a halo (19). Histopathological characteristics include granulation tissue located around the 
respiratory bronchioles and within the alveoli, or polypoid tissues composed of fibroblasts. Macroscopically, these may have a patchy, strip-like distribution. Mucinous fibroblast nodules known as Masson bodies may be observed in the bronchial cavities, alveolar ducts and alveolar cavities (20). These oval-or polygonal-shaped bodies rich in mucopolysaccharides are known as a hallmark pathological sign of COP.

Biomarkers including Krebs von den Lungen-6 (KL-6), tenascin $\mathrm{C}$ and galectin-9 in bronchoalveolar lavage fluid may aid in the diagnosis of COP, but with certain limitations $(21,22)$. At present, a definitive diagnosis of COP requires either open or thoracoscopic lung biopsy. The current clinical treatment of COP is still based on corticosteroid therapy, and $65-80 \%$ of patients with COP achieve complete clinical and pathological remission after corticosteroid therapy. Although COP responds well to corticosteroids, it may be prone to relapse following treatment $(23,24)$. COP may be induced by numerous factors, including viral infections and autoimmune diseases, but its pathogenesis remains incompletely understood and warrants further study.

HT is an organ-specific autoimmune disease. In the present case, the thyroid gland revealed diffuse enlargement with nodular changes of the gland surface. On thyroid biopsy, a large number of dendritic cells was observed, which is noteworthy, as in the presence of HT lesions, dendritic cells may activate immune cells and cause immune-mediated injury (8). Elevation of TPOAb and TgAb levels is sensitive and specific for the diagnosis of HT. Diffuse goitre, particularly with vertebral lobe enlargement of the isthmus, or changes in thyroid function, may indicate HT. If serum $\mathrm{TPOAb}$ and $\mathrm{Tg} \mathrm{Ab}$ are significantly increased, the diagnosis may be confirmed. Following a diagnosis of HT, one should pay attention to the emergence of thyroid tumours and clinical hypothyroidism (8-11). At present, the treatment of HT is based replacement of deficient innate thyroid hormone production with levothyroxine, an exogenous thyroid hormone.

In the patient of the present study, pneumonia was considered as the likely diagnosis at the local hospital early in the onset of the disease, but no significant improvement was observed after antibiotic treatment. However, in the early stages of the disease, the patient exhibited fever, chest imaging changes and a slight elevation of infection indicators, including white blood cell count, all consistent with a diagnosis of pneumonia. Severe pneumonia was still considered the likely diagnosis upon transfer to the intensive care unit, but no significant improvement was achieved after broad-spectrum anti-microbial treatment, and the abnormalities on chest imaging did not improve. No causative pathogens were detected after broncheolar lavage. . Bronchoscopy and lung biopsy combined with imaging finally led to the diagnosis of COP. At the same time, the combination of thyroid function test and thyroid ultrasonography confirmed COP present concomitantly with HT, although the patient's thyroid function was normal and no thyroid tumour was observed. Therefore, antibiotics were discontinued and the patient was treated with methylprednisolone. After treatment, the patient's shortness of breath resolved and the non-invasive ventilator was completely disengaged. Chest imaging revealed that the lung consolidations gradually resolved over a period of 6 months after treatment, at which point it was indicated that the condition was completely controlled. After 12 months of follow-up, the lung imaging was completely normal, pulmonary function was completely normal and no recurrence was observed. The thyroid examination was normal, the patient was generally in good health and her activities of daily life were not affected. The two diseases discussed in the present study may be induced by autoimmune factors. The patient's relevant laboratory examinations indicated a weakly positive anti-nuclear antibody ratio (1:80) and a low CD4/CD8 cell ratio, giving rise to the speculation that autoimmune factors may have been causative of these two conditions. HT may lead to or exacerbate COP and the two diseases may clearly coexist, as demonstrated in this patient. Such coexistence of HT and COP in the same patient has rarely been reported in the literature (13). The diagnosis and treatment of this case are reported in the present study and hopefully, clinicians will take note.

In short, when patients with pulmonary exudation and consolidation appear in clinical practice and prove unresponsive to the conventional anti-infective treatment, a differential diagnosis should be considered. Bronchoscopy or lung biopsy are recommended to confirm the diagnosis. It is important to identify whether associated diseases including HT accompany $\mathrm{COP}$ in order to reduce misdiagnosis and improve the cure rate and quality of life of patients.

\section{Acknowledgements}

Not applicable.

\section{Funding}

No funding was received.

\section{Availability of data and materials}

All data generated or analyzed during the present study are included in this published article.

\section{Authors' contributions}

LG designed the study, collected and analysed the data, selected the patient for the study and wrote the manuscript. $\mathrm{BC}$ collected and analysed the data and revised the manuscript. LZ collected the data and helped select the patient for the study. YD designed the study and collected the data. HL collected and analysed the patient data and revised the manuscript. QS designed the study, collected the data and wrote the manuscript.

\section{Ethics approval and consent to participate}

The present study was performed in accordance with the Declaration of Helsinki. Informed consent was obtained from the patient of the present study.

\section{Patient consent for publication}

The patient consented to the publication of her images and data in this case report. 


\section{Competing interests}

The authors declare that they have no competing interests.

\section{References}

1. Kim AR, Yoo KH, Lee KY, Kim SJ, Kim HJ, Kim JH and Rhyu YA: A case of cryptogenic organizing pneumonia after transarterial chemoembolization for the treatment of hepatocellular carcinoma. Tuberc Respir Dis (Seoul) 78: 469-472, 2015.

2. Izykowski N, Kuehnel M, Hussein K, Mitschke K, Gunn M, Janciauskiene S, Haverich A, Warnecke G, Laenger F, Maus U and Jonigk D: Organizing pneumonia in mice and men. J Transl Med 14: 169-180, 2016

3. Davison AG, Heard BE, McAllister WA and Turner-Warwick ME: Cryptogenic organizing pneumonitis. Q J Med 52: 382-394, 1983

4. Travis WD, Costabel U, Hansell DM, King TE Jr, Lynch DA, Nicholson AG, Ryerson CJ, Ryu JH, Selman M, Wells AU, et al: An official American Thoracic Society/European respiratory society statement: Update of the international multidisciplinary classification of the idiopathic interstitial pneumonias. Am J Respir Crit Care Med 188: 733-748, 2013.

5. D'Aurizio F, Villalta D, Metus P, Doretto P and Tozzoli R: Is vitamin $\mathrm{D}$ a player or not in the pathophysiology of autoimmune thyroid diseases? Autoimmun Rev 14: 363-369, 2014.

6. Banga JP and Schott M: Autoimmune thyroid diseases. Int J Dermatol 47: 699-701, 2015.

7. Hashimoto H: Zur Kenntniss der lymphomatosen Veranderung der Schilddruse (Struma lymphomatosa). Arch Klin Chir 97: 219-248, 1912.

8. Silva de Morais N, Stuart J, Guan H, Wang Z, Cibas ES, Frates MC, Benson CB, Cho NL, Nehs MA, Alexander CA, et al: The impact of hashimoto thyroiditis on thyroid nodule cytology and risk of thyroid cancer. J Endocr Soc 3: 791-800, 2019.

9. Chen YK, Lin CL, Cheng FT, Sung FC and Kao CH: Cancer risk in patients with Hashimoto's thyroiditis: A nationwide cohort study. Br J Cancer 109: 2496-2501, 2013.

10. Kim SH, Choi JY and Yun JS: Features of papillary thyroid micro carcinoma in the presence and absence of lymphocytic thyroiditis. Endocr Pathol 21: 149-153, 2010.

11. Bedir R and Yilmaz R: Coexistence of papillary thyroid cancer and Hashimoto's thyroiditis developing within an ovarian mature cystic teratoma. J Midlife Health 10: 45-47, 2019.

12. Hwang YC, Kim TY, Kim WB, Shong YK, Yi KH, Shong M, Jo YS, Kim WS and Chung JH: Clinical characteristics of primary thyroid lymphoma in Koreans. Endoer J 56: 399-405, 2009.

13. Radzikowska E, Wiatr E, Remiszewski P, Bestry I, Grudny J, Langfort R, Szopiński J,Zych J,Płodziszewska M,Rudziński P, et al: Organizing pneumonia-analysis of 18 own cases. Pneumonol Alergol Pol 72: 99-104, 2004 (In Polish).
14. Kligerman SJ, Franks TJ and Galvin JR: From the radiologic pathology archives: Organization and fibrosis as a response to lung injury in diffuse alveolar damage, organizing pneumonia, and acute fibrinous and organizing pneumonia. RadioGraphics 33: 1951-1975, 2013.

15. Pardo J, Panizo A, Sola I, Queipo F, Martinez-Peñuela A and Carias R: Prognostic value of clinical, morphologic, and immunohistochemical factors in patients with bronchiolitis obliterans-organizing pneumonia. Hum Pathol 44: 718-724, 2013

16. Niksarlıoğlu EY, Özkan GZ, Bakan ND, Yurt S, Kılıç L and Çamsarı G: Cryptogenic organizing pneumonia: Clinical and radiological features, treatment outcomes of 17 patients, and review of the literature. Turk J Med Sci 46: 1712-1718, 2016.

17. Taniguchi $\mathrm{H}$ and Kondoh $\mathrm{Y}$ : Acute and subacute idiopathic interstitial pneumonias. Respirology 21: 810-820, 2016.

18. Yılmaz S, Akıncı Özyürek B, Erdoğan Y, Cirit Koçer B, Demirağ F, Dadalı Y and Büyükyaylacı Özden S: Retrospective evaluation of patients with organizing pneumonia: Is cryptogenic organizing pneumonia different from secondary organizing pneumonia? Tuberk Toraks 65: 1-8, 2017.

19. Chung MP, Nam BD, Lee KS, Han J, Park JS, Hwang JH, Cha MJ and Kim TJ: Serial chest CT in cryptogenic organizing pneumonia: Evolutional changes and prognostic determinants. Respirology 23: 325-330, 2018.

20. Shen L, Liu J, Huang L, Zhang Y, Xiao X and Yu H: Cryptogenic organizing pneumonia presenting as a solitary mass: clinical, imaging, and pathologic features. Med Sci Monit 25: 466-474, 2019.

21. Radzikowska E, Roży A, Jaguś P, Wiatr E, Gawryluk D, Chorostowska-Wynimko J and Roszkowski-Śliż K: Cryptogenic organizing pneumonia: IL- $1 \beta$, IL- 6 , IL- 8 , and TGF- $\beta 1$ serum concentrations and response to clarithromycin treatment. Adv Exp Med Biol 911: 77-85, 2016

22. Katoh S, Ikeda M, Shimizu H, Abe M, Ohue Y, Mouri K, Kobashi Y and Oka M: Increased Galectin-9 concentration and number of CD4+Foxp3high+cells in bronchoalveolar lavage fluid of patients with cryptogenic organizing pneumonia. Lung 193: 683-689, 2015.

23. Zhou Y, Wang L, Huang M, Ding J, Jiang H, Zhou K, Meng F, Xiao Y, Cai H and Dai J: A long-term retrospective study of patients with biopsy-proven cryptogenic organizing pneumonia. Chron Respir Dis 16: 1479973119853829, 2019.

24. Lebargy F, Picard D, Hagenburg J, Toubas O, Perotin JM, Sandu S, Deslee G, Dury S: Micronodular pattern of organizing pneumonia: Case report and systematic literature review. Medicine (Baltimore) 96: e5788, 2017.

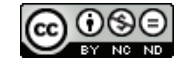

This work is licensed under a Creative Commons Attribution-NonCommercial-NoDerivatives 4.0 International (CC BY-NC-ND 4.0) License. 